proposed in the management of pulmonary artery sarcoma or thromboembolic disease.

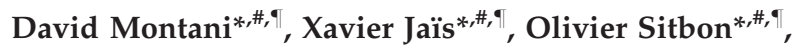 Philippe Dartevelle ${ }^{+}$, Gérald Simonneau ${ }^{*, \#, \oplus}$ and Marc Humbert $*, \#$,}

*Faculté de Médecine, Université Paris-Sud, "Assistance Publique Hôpitaux de Paris, Service de Pneumologie et Réanimation Respiratoire, Hôpital Bicêtre, Le KremlinBicêtre, "INSERM U999 LabEX LERMIT, and ${ }^{+}$Service de Chirurgie Thoracique, Centre Chirurgical Marie Lannelongue, Le Plessis-Robinson, France.

Correspondence: D. Montani, Service de Pneumologie et Réanimation Respiratoire, Hôpital Bicêtre, 78, rue du Général Leclerc, Le Kremlin-Bicêtre, 94270, France. E-mail: david.montani @abc.aphp.fr

Statement of Interest: Statements of interest for all authors of this manuscript can be found at www.erj.ersjournals.com/site/misc/ statements.xhtml

\section{REFERENCES}

1 Park JS, Chung J-H, Jheon S. EBUS-TBNA in the differential diagnosis of pulmonary artery sarcoma and thromboembolism. Eur Respir J 2011; 38: 1480-1482.

2 Blackmon SH, Rice DC, Correa AM, et al. Management of primary pulmonary artery sarcomas. Ann Thorac Surg 2009; 87: 977-984.

3 Cox JE, Chiles C, Aquino SL, et al. Pulmonary artery sarcomas: a review of clinical and radiologic features. J Comput Assist Tomogr 1997; 21: 750-755.

4 Ito K, Kubota K, Morooka M, et al. Diagnostic usefulness of ${ }^{18}$ F-FDG $\mathrm{PET} / \mathrm{CT}$ in the differentiation of pulmonary artery sarcoma and pulmonary embolism. Ann Nucl Med 2009; 23: 671-676.

5 Chong S, Kim TS, Kim BT, et al. Pulmonary artery sarcoma mimicking pulmonary thromboembolism: integrated FDG PET/ CT. AJR Am J Roentgenol 2007; 188: 1691-1693.

6 Mayer E, Jenkins D, Lindner J, et al. Surgical management and outcome of patients with chronic thromboembolic pulmonary hypertension: results from an international prospective registry. J Thorac Cardiovasc Surg 2011; 141: 702-710.

7 Winchester PA, Khilnani NM, Trost DW, et al. Endovascular catheter biopsy of a pulmonary artery sarcoma. AJR Am J Roentgenol 1996; 167: $657-659$

\title{
Nomogram to predict the presence of EGFR activating mutation in lung adenocarcinoma
}

\section{To the Editors:}

GIRARD et al. [1] developed a nomogram to predict the presence of epidermal growth factor receptor (EGFR) activating mutations. Before using it in practice, however, the precision and limitations must be considered. Patient selection for tyrosine kinase inhibitors (TKIs) based exclusively on EGFR mutations is still controversial, because clinical response rates to TKI are nearly $70 \%$ in tumours harbouring EGFR activating mutations. Other factors, such as amplification of the EGFR gene and the activity of molecules downstream of EGFR, such as phosphorylated protein kinase B, and mutations of KRAS can play a role in the sensitivity to EGFR inhibitors. Again, $10-20 \%$ of patients with a partial response to gefitinib did not have identifiable EGFR mutations, signifying that EGFR mutations are not the only determinants of TKI response [2]. The incidence of EGFR mutations in East Asia is nearly $40 \%$, compared with $10 \%$ in Europe and the USA. The prediction accuracy of the nomogram for EGFR mutation is unsatisfactory as the concordance index is low (0.64) in the East-Asian population, where the need for reliable predictive nomograms is higher [1].

Differences in all the above factors and population characteristics can challenge the accuracy and concordance of nomograms when they are applied to an Asian/non-Asian population. Before a nomograms is put to widespread use, it needs external independent validation. Despite external validitation, we do not know whether the existing evidence supports the impact of nomograms on medical decision making. There are no randomised studies that clearly reveal that the use of prediction nomograms improves decision making or patient care, reduces patient anxiety and is harmless.

In Chinese nonsmall cell lung cancer patients, the EGFR mutation status determined in serum DNA using mutantenriched sequencing corresponds to that demonstrated in paired tumour tissues (concordance rate of 93.1\%) suggesting that serum DNA is a practical and reliable source of tumour DNA for detecting EGFR mutations [3]. This concordance rate (93.1) is higher than the nomogram's concordance index of 0.84 and 0.64 in non-Asian and East-Asian populations, respectively. Another study confirmed high sensitivity (92\%) and specificity $(100 \%)$ of plasma EGFR mutation analysis by microfluidics digital PCR [4]. If we are unable to obtain tumour tissue or block, where diagnosis is based only on needle aspiration or cytology, it is practicable now to use serum DNA to detect EGFR mutation status and to evaluate its potential as a predictor of response to EGFR-TKIs without waiting for biopsy tissue.

In this scenario, nomograms can be built to predict the treatment outcome of TKIs. Predictive models can help 
clinicians not only to plan treatments but also to inform patients about the options and potential consequences of therapies in better ways. This can help to involve patients in the decision-making process regarding management and can improve compliance.

\section{Prasanta Raghab Mohapatra*, Sachin Punatar" and Kumar Prabhash ${ }^{\#}$ \\ *Government Medical College and Hospital, Chandigarh, and \\ ${ }^{\#}$ Tata Memorial Hospital, Mumbai, India.}

Correspondence: P.R. Mohapatra, Government Medical College and Hospital, Sector-32, Chandigarh, India. E-mail: prmohapatra@hotmail.com

Statement of Interest: None declared.

\section{REFERENCES}

1 Girard N, Sima CS, Jackman DM, et al. Nomogram to predict the presence of EGFR activating mutation in lung adenocarcinoma. Eur Respir J 2012; 39: 366-372.

2 Sharma SV, Bell DW, Settleman J, et al. Epidermal growth factor receptor mutations in lung cancer. Nat Rev Cancer 2007; 7: 169-181.

3 Jiang B, Liu F, Yang L, et al. Serum detection of epidermal growth factor receptor gene mutations using mutant-enriched sequencing in Chinese patients with advanced non-small cell lung cancer. J Int Med Res 2011; 39: 1392-1401.

4 Yung TK, Chan KC, Mok TS, et al. Single-molecule detection of epidermal growth factor receptor mutations in plasma by microfluidics digital PCR in non-small cell lung cancer patients. Clin Cancer Res 2009; 15: 2076-2084.

DOI: $10.1183 / 09031936.00022112$

\section{From the authors:}

We thank P. Mohapatra and co-workers for their interest in our article. Their comments highlight some of the current challenges in predicting response to epidermal growth factor receptor (EGFR) tyrosine kinase inhibitors (TKIs) in lung cancer. We built our nomogram to predict the presence of EGFR activating mutations, as these alterations represent the best available predictive biomarker in this setting, at a time when data about tumour response to EGFR TKIs were not available from large cohorts of patients [1]. Once such data emerge, a predictive model of tumour response to EGFR TKIs, as well as other targeted therapies in other molecular subsets of lung tumours, may be developed.

As we stated, EGFR genotyping is now a standard, and should be obtained when possible. Physicians, as well as healthcare providers, need to work on offering each patient with lung cancer molecular diagnoses, including EGFR mutation genotyping, as well as testing for other oncogenic alterations if specific targeted therapies are available [2]. Despite undeniable progress in recent years, one must keep in mind that molecular diagnoses are not routinely available in numerous countries, including European countries.
Mutation genotyping based on serum tumour DNA or circulating tumour cells is a promising concept, and may be useful when access to tumour tissue is limited [3,4]. However, such techniques remain to be evaluated and implemented in routine practice, even in developed countries. Of note, the two papers cited by P. Mohapatra and co-workers included only 58 and 35 patients, respectively [3,4]. Ultimately, the evaluation of such new predictive biomarkers is challenging, and beyond the small experience reported so far, dedicated prospective trials may be required to accurately assess their sensitivity, specificity and positive and negative predictive values.

In the end, nomograms are user-friendly graphical representations of predictive models. Nomograms have been evaluated in specific clinical situations, and subsequently integrated in clinical practice guidelines, especially in prostate cancer $[5,6]$. As we discussed in our article, our nomogram, despite its strong accuracy in non-Asian patients, does have some limitations. Any prediction instrument incorporates a certain degree of uncertainty. Although we built our model using an international multicentre cohort of patients, our nomogram needs to be further validated in additional cohorts. Its potential role in individual decision making remains to be determined. Aiming at facilitating subsequent studies, we have developed a website (www.myEGFRscore.com) that, based on our predictive model, provides the individual probability of EGFR mutation in non-Asian patients with lung adenocarcinoma tumours, in an even more straightforward way in routine clinical care.

\section{Nicolas Girard}

Hospices Civils de Lyon, Lyon, France and Université Claude Bernard Lyon 1, Lyon, France.

Correspondence: N. Girard, Service de Pneumologie, Hôpital Louis Pradel, 28 avenue doyen Lépine, 69500 Bron, France. E-mail: nicolas.girard@chu-lyon.fr

Statement of Interest: None declared.

\section{REFERENCES}

1 Girard N, Sima CS, Jackman DM, et al. Nomogram to predict the presence of EGFR activating mutation in lung adenocarcinoma. Eur Respir J 2012; 39: 366-372.

2 Pao W, Girard N. New driver mutations in non-small-cell lung cancer. Lancet Oncol 2011; 12: 175-180.

3 Jiang B, Liu F, Yang L, et al. Serum detection of epidermal growth factor receptor gene mutations using mutant-enriched sequencing in Chinese patients with advanced non-small cell lung cancer. J Int Med Res 2011; 39: 1392-1401.

4 Yung TK, Chan KC, Mok TS, et al. Single-molecule detection of epidermal growth factor receptor mutations in plasma by microfluidics digital PCR in non-small cell lung cancer patients. Clin Cancer Res 2009; 15: 2076-2084.

5 Lecuona A, Heyns CF. A prospective, randomized trial comparing the Vienna nomogram to an eight-core prostate biopsy protocol. BJU Int 2011; 108: 204-108.

6 Susman E, Phend C. Nomograms inserted into NCCN prostate cancer guidelines. Oncology Times 2003; 25: 28-31. 